American J. of Engineering and Applied Sciences 4 (1): 61-65, 2011

ISSN 1941-7020

(C) 2010 Science Publications

\title{
Optical Properties of Crosslinked Chitosan Thin Film with Glutaraldehyde Using Surface Plasmon Resonance Technique
}

\author{
${ }^{1}$ Yap Wing Fen, ${ }^{1}$ W. Mahmood Mat Yunus, ${ }^{1}$ Mohd Maarof Moksin, \\ ${ }^{1}$ Zainal Abidin Talib and ${ }^{2}$ Nor Azah Yusof \\ ${ }^{1}$ Department of Physics, \\ ${ }^{2}$ Department of Chemistry \\ Faculty of Science, University Putra Malaysia, \\ 43400 UPM Serdang, Selangor, Malaysia
}

\begin{abstract}
Problem statement: The aim of this research is to study the optical properties of crosslinked chitosan thin film with glutaraldehyde using Surface Plasmon Resonance (SPR) technique. Approach: The crosslinked chitosan is synthesized by homogeneous reaction of medium molecular weight chitosan in aqueous acetic acid solution with glutaraldehyde as crosslinking agent. Four different amount of chitosan, i.e., 0.40, 0.50, 0.60 and $0.70 \mathrm{~g}$ of chitosan, were dissoloved in 50 $\mathrm{ml}$ acetic acid separately. Glass cover slips (as substrates) were first coated with two sets of different thickness of gold layer. Then the amount of $0.55 \mathrm{~mL}$ of each crosslinked chitosan solution was spin coated onto the gold layer. Results: Using developed multilayer Matlab fitting program (matrix method based), the experimental surface plasmon resonance curves were fitted to obtain the thickness and refractive index of crosslinked chitosan thin film. The real and imaginary part of refractive index of crosslinked chitosan thin film are $(1.540 \pm 0.005)$ and $(0.015 \pm 0.002)$, respectively. Conclusion: The results are similar for all different thickness of the thin films and not affected by the thickness of gold layer. This study is important for future research on multilayer SPR sensor.
\end{abstract}

Key words: Surface plasmon resonance, crosslinked chitosan, glucosidic bonds, plasmon resonance technique, homogeneous reaction

\section{INTRODUCTION}

Chitosan is a copolymer of glucosamine and $\mathrm{N}$ acetyl glucosamine linked by $\beta-1,4$ glucosidic bonds. Chitosan occurs naturally in some microorganisms, yeast and fungi. It is a non-toxic, biocompatible and biodegradable natural polymer. The commercially available chitosan is mostly derived by alkaline $\mathrm{N}$ deacetylation from chitin of crustaceans because it is easily obtained from the shells of crabs, shrimps, lobsters and krill. These two low-cost natural materials had been used for absorption of metal ions, dyes and protein. Compared to chitin, chitosan is more efficient in absorption capacity due to the presence of a large number of amino groups on chitosan chain. However, chitosan is soluble in organic acid, such as acetic acid and formic acid (Shauer et al., 2003).

Crosslinking is an important step to improve the chemical stability of chitosan (Bhumkar and Pokharkar, 2006). One crosslinking agent, glutaraldehyde, is an organic compound with the formula $\mathrm{CH}_{2} \quad\left(\mathrm{CH}_{2} \mathrm{CHO}\right)_{2}$. Glutaraldehyde is frequently used in biochemistry applications as an amine-reactive homobifunctional crosslinker.

Surface Plasmon Resonance (SPR) spectroscopy is a surface-sensitive technique that has been used to characterize the thickness and refraction index of dielectric medium at noble metal surface (Yusmawati et al., 2007). For the last decade, surface plasmon resonance sensors have been extensively studied. SPR technique has emerged as a powerful technique for a variety of chemical and biological sensor applications. The first chemical sensing based on SPR technique was reported by Liedberg et al. (1983). SPR is an optical process in which light satisfying a resonance condition excites a charge-density wave propagating along the interface between a metal and dielectric material by monochromatic and p-polarized light beam. The intensity of the reflected light is reduced at a specific incident angle producing a sharp shadow (called surface plasmon resonance) due to the resonance energy o ccurs between the incident beam and surface plasmon

Corresponding Author: Yap Wing Fen, Department of Physics, Faculty of Science, University Putra Malaysia, 43400 UPM Serdang, Selangor, Malaysia 
wave (Homola et al., 1999). SPR is regarded as a simple optical technique for surface and interfacial studies and shows the great potential for investigating biomolecules (Homola, 2006). SPR has been widely demonstrated as an effective optical technique for the study of interfaces and thin films (Mendelez et al., 1997).

Due to the chelating ability of chitosan, we are interested to investigate the optical properties of crosslinked chitosan film by using SPR.

\section{MATERIALS AND METHODS}

Chitosan with medium molecular weight and degree of deacetylation $75-85 \%$ was purchased from Sigma Aldrich (St. Louis, MO, USA). Acetic acid and glutaraldehyde were also obtained from Aldrich. Prism with refractive index, $\mathrm{n}=1.77861$ at $632.8 \mathrm{~nm}$ and the substrate, glass cover slips $24 \times 24 \mathrm{~mm}$ with thickness 0.13-0.16 mm were purchased from Menzel-Glaser.

To prepare chitosan solution, $0.40,0.50,0.60$ and $0.70 \mathrm{~g}$ of chitosan were weighed separately. Then each of the samples was dissolved in $50 \mathrm{~mL} 1 \%$ acetic acid in four different beakers. The samples were stirred for $24 \mathrm{~h}$ until all the chitosan was dissolved in acetic acid. Then glutaraldehyde was added to the solution to crosslink chitosan. The cross-link ratio of glutaraldehyde with chitosan was about 0.13 . The resulting samples were stirred for another $1 \mathrm{~h}$.

The glass cover slips were cleaned using acetone to cleanse off the dirt or remove fingerprint marks laid on the surface of glass slides. Then they were deposited with gold layer using SC7640 Sputter Coater with Film Thickness Monitor (FTM) at 33 and 38 for two different thicknesses of gold layers.

Spin coating technique was used to produce a thin layer of chitosan film on the top of the gold layer. Approximately $0.55 \mathrm{~mL}$ of the solution was placed on the glass cover slip covering the majority of the surface. The glass cover slip were spun at $6000 \mathrm{rev} \mathrm{min}^{-1}$ for 30 sec using Spin Coating System, P-6708D.

Figure 1 shows the experimental setup for SPR measurement. The SPR measurement had been carried out by measuring the reflected HE-Ne laser beam $(632.8 \mathrm{~nm}, 5 \mathrm{~mW})$ as a function of incident angle. The optical set up consists of a He-Ne laser, an optical stage driven by a stepper motor with a resolution of 0.0010 (Newport MM 3000), a light attenuator, a polarizer and an optical chopper (SR 540). The reflected beam was detected by a sensitive photodiode and then processed by the lock-in-amplifier (SR 530).

The coated glass cover slip was attached to the prism by using index matching liquid. The prism with

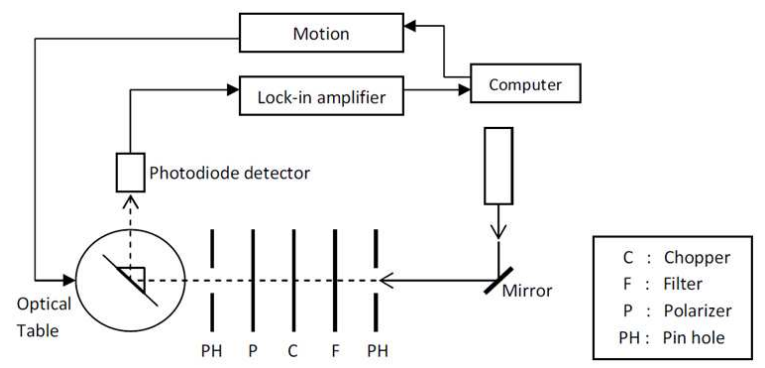

Fig. 1: Experimental setup for angle scan surface plasmon resonance technique

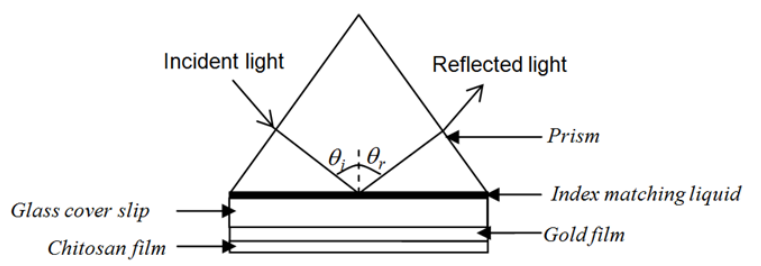

Fig. 2: Arrangement of the prism and glass cover slip for SPR measurement

glass cover slip was mounted on a rotating plate to control the angle of the incident light. Figure 2 shows the arrangement of the prism and glass cover slip for SPR measurement (Kurihara and Suzuki, 2002).

\section{RESULTS}

For the first set of thin film (coated with gold at FTM 33), the preliminary SPR test was carried out for gold film contact with air (single layer) to determine the optical properties of gold layer, i.e., the real part refractive index, $n$; the imaginary part of refractive index, $k$; the real part of permittivity, $\varepsilon_{r}$; the imaginary part of permittivity, $\varepsilon_{i}$; and the thickness, $d$ of the thin film. The SPR curve was obtained and fitted as shown in Fig. 3. We obtained the optical properties of gold layer by using the developed Matlab fitting program (matrix method). The values of refractive index, $n$ and $k$, for gold layer are 0.188 and 3.201 respectively; the dielectric constants, $\varepsilon_{r}$ and $\varepsilon_{i}$, are 10.211 and 1.204 respectively, while the thickness, $d$ is $30.9 \mathrm{~nm}$. This information is important for the further fitting for multilayer.

Then, the SPR experiment was carried out for gold/chitosan film contact with air and the SPR curves were obtained and fitted as shown in Fig. 4a and 4b. Using the multilayer Matlab fitting program, the properties of crosslinked chitosan thin film was determined. The properties of gold layer obtained from 


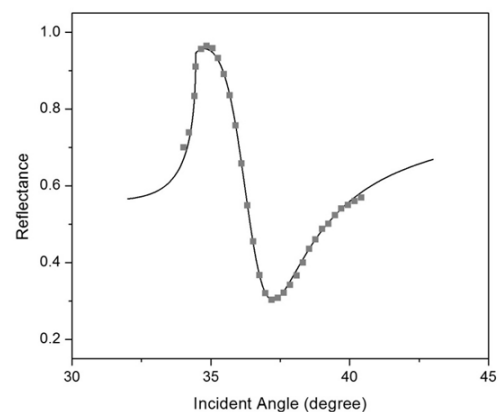

Fig. 3: The SPR curve for gold film (FTM 33) in contact with air. The solid line represents the theoretical curve

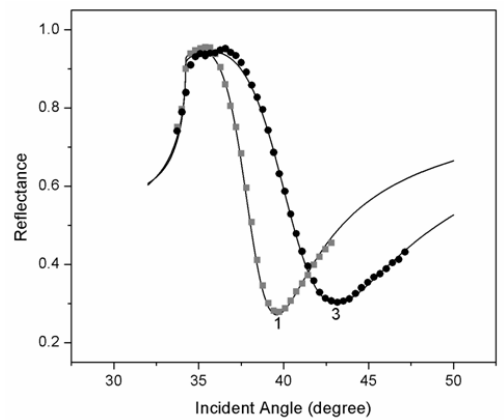

(a)

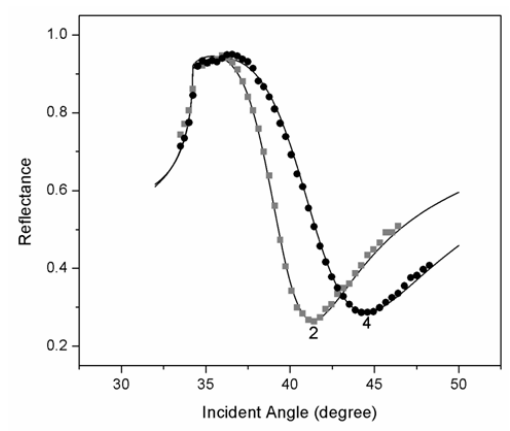

(b)

Fig. 4: The SPR curve for gold/chitosan film contact with air: different amount of chitosan, i.e. (1) $0.40 \mathrm{~g}$ (2) $0.50 \mathrm{~g}$ (3) $0.60 \mathrm{~g}$ and (4) $0.70 \mathrm{~g}$, in acetic acid coated on the same thickness of gold film (FTM 33). The solid line represents the theoretical curve

earlier fitting, i.e., real part of refractive index $(0.188 \pm$ 0.005 ), imaginary part of refractive index (3.201 \pm $0.002)$ and thickness $(30.9 \pm 0.1) \mathrm{nm}$, was used in this multilayer fitting process. From the fitting, the thickness of the crosslinked chitosan film as well as the real and imaginary part of refractive index are for all

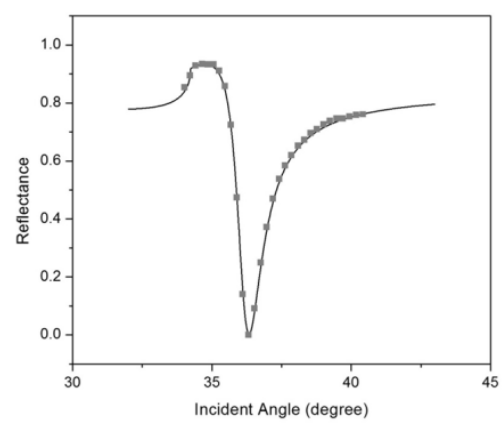

Fig. 5: The SPR curve for gold film (FTM 38) in contact with air. The solid line represents the theoretical curve

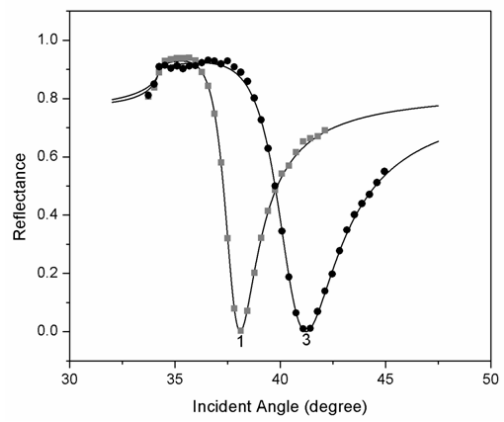

(a)

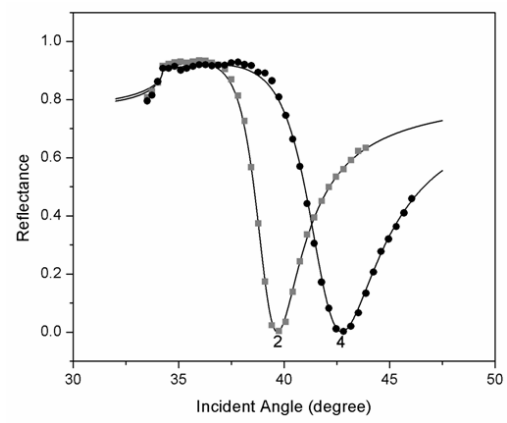

(b)

Fig. 6: The SPR curve for gold/chitosan film contact with air: different amount of chitosan, i.e. (1) $0.40 \mathrm{~g}$ (2) $0.50 \mathrm{~g}$ (3) $0.60 \mathrm{~g}$ and (4) $0.70 \mathrm{~g}$, in acetic acid coated on the same thickness of second set gold film (FTM 38). The solid line represents the theoretical curve

four concentrations obtained and tabulated as shown in Table 1. Table 2 shows the resonance angle and the shift of resonance angle for different concentration of chitosan in acetic acid.

The preliminary SPR test was also carried out for the second set of gold film (coated at FTM 38) which 
Am. J. Engg. \& Applied Sci., 4 (1): 61-65, 2011

Table 1: The thickness of crosslinked chitosan thin film, the real and imaginary part of refractive index for different concentration of chitosan in acetic acid (for set 1 gold film)

\begin{tabular}{llll}
\hline $\begin{array}{l}\text { Concentration of } \\
\text { chitosan in acetic } \\
\text { acid }(\mathrm{g})\end{array}$ & $\begin{array}{l}\text { Thickness of } \\
\text { chitosan } \\
\text { film, } d(\mathrm{~nm})\end{array}$ & $\begin{array}{l}\text { Real part of } \\
\text { refractive } \\
\text { index, } n\end{array}$ & $\begin{array}{l}\text { Imaginary part of } \\
\text { refractive index, } k\end{array}$ \\
\hline 0.40 & 15 & 1.540 & 0.015 \\
0.50 & 25 & 1.540 & 0.015 \\
0.60 & 31 & 1.540 & 0.014 \\
0.70 & 36 & 1.540 & 0.015 \\
\hline
\end{tabular}

Table 2: The SPR resonance angle and shift of resonance angle for different concentration of chitosan in acetic acid (for set 1 gold film)

\begin{tabular}{lll}
\hline $\begin{array}{l}\text { Concentration of chitosan } \\
\text { in acetic acid }(\mathrm{g})\end{array}$ & $\begin{array}{c}\text { Resonance Angle, } \theta \text { min } \\
\text { (degree) }\end{array}$ & $\begin{array}{l}\text { Shift of Resonance } \\
\text { Angle, } \Delta \theta \text { (degree) }\end{array}$ \\
\hline 0.4 & 39.589 & 2.359 \\
0.5 & 41.86 & 4.63 \\
0.6 & 43.092 & 5.862 \\
0.7 & 44.497 & 7.267 \\
\hline
\end{tabular}

Table 3: The thickness of crosslinked chitosan thin film, the real and imaginary part of refractive index for different concentration of chitosan in acetic acid (for set 2 gold film)

\begin{tabular}{llll}
\hline $\begin{array}{l}\text { Concentration of } \\
\text { chitosan in } \\
\text { acetic acid }(\mathrm{g})\end{array}$ & $\begin{array}{l}\text { Thickness of } \\
\text { chitosan film, } \\
d(\mathrm{~nm})\end{array}$ & $\begin{array}{l}\text { Real part of } \\
\text { refractive index, } n\end{array}$ & $\begin{array}{l}\text { Imaginary part of } \\
\text { refractive index, } k\end{array}$ \\
\hline 0.40 & 14 & 1.540 & 0.015 \\
0.50 & 23 & 1.540 & 0.014 \\
0.60 & 30 & 1.540 & 0.016 \\
0.70 & 36 & 1.540 & 0.015 \\
\hline
\end{tabular}

Table 4: The SPR resonance angle and shift of resonance angle for different concentration of chitosan in acetic acid (for set 2 gold film)

\begin{tabular}{lll}
\hline $\begin{array}{l}\text { Concentration of chitosan } \\
\text { in acetic acid }(\mathrm{g})\end{array}$ & $\begin{array}{l}\text { Resonance Angle, } \theta_{\min } \\
\text { (degree) }\end{array}$ & $\begin{array}{l}\text { Shift of Resonance } \\
\text { Angle, } \Delta \theta \text { (degree) }\end{array}$ \\
\hline 0.4 & 38.093 & 1.762 \\
0.5 & 39.677 & 3.346 \\
0.6 & 41.218 & 4.887 \\
0.7 & 42.732 & 6.401 \\
\hline
\end{tabular}

contact with air to determine the properties of gold layer. The SPR curve was obtained and fitted as shown in Fig. 5. Also using the developed Matlab fitting program, the optical properties, $n, k, \varepsilon_{r}$ and $\varepsilon_{i}$, for gold layer are obtained; i.e., $0.190,3.305,-10.887$ and 1.256 respectively; while $\mathrm{d}$ of the gold layer is $47.3 \mathrm{~nm}$.

Then, the SPR experiment was carried out for gold/chitosan film contact with air and the SPR curves were obtained as shown in Fig. 6a and 6b. The optical properties of gold layer obtained from earlier fitting, i.e., $n(0.190 \pm 0.005), k(3.305 \pm 0.002)$ and $d(47.3 \pm$ $0.1) \mathrm{nm}$, was used for multilayer fitting to characterize chitosan film. From the fitting, the thickness of the crosslinked chitosan thin film and also the real and imaginary part of refractive index are for all four concentrations obtained and tabulated as shown in
Table 3. Table 4 shows the resonance angle and the shift of resonance angle for different concentration of chitosan in acetic acid.

\section{DISCUSSION}

The fitting result for gold layer (single layer) shows a good agreement reported by Sadowski et al. (1994). There is only a small difference for the refractive index and the permittivity between the two thicknesses of gold layer. We believe that it depends on the various deposition parameters such as deposition rate, vacuum pressure and substrate temperature as reported by Berger et al. (1992). The optical properties for first set of gold film which is thinner gold film have larger deviation. The very thin $(\mathrm{d} \approx 30 \mathrm{~nm})$ metal films are usually discontinuous, showing changes especially in the optical permittivity and refractive index. These inhomogeneities which lie close enough to the film surface and known as surface roughness, opens an additional channel for the radiative decay of plasmons resulting in light emission to the air side of the film (Hoffmann et al., 1996).

From the results, we found that the refractive index of crosslinked chitosan thin film for both thicknesses of gold layer and all concentrations of chitosan is similar, i.e., real part $(1.540 \pm 0.005)$ and imaginary part $(0.015$ \pm 0.002 ). Hence we proved that the refractive index of the crosslinked chitosan thin film is not depending on the thickness of the thin film formed. Also, the thickness of the gold film is not affected the optical properties of crosslinked chitosan thin film.

From Fig. 4 and 6, we observed that the resonance angle shifted more to right as amount of chitosan in acetic acid increased. It means the resonance angle increased as the thickness of the chitosan thin film increased. On the other hand, the width and asymmetry of the reflectivity dip increase with the same trend. The dip produced at curve 1 is the narrowest followed by curve 2, 3 and 4 .

\section{CONCLUSION}

In this study, the optical properties of crosslinked chitosan thin film with glutaraldehyde have been obtained using surface plasmon resonance technique. The resonance angle shifted to higher values to the right with the increasing of the concentration of chitosan in acetic acid. It means the change of resonance angle $(\Delta \theta)$ increased as the thickness of the chitosan film increased. However, the optical properties of chitosan film are not affected by the thickness of the film. The refractive index of crosslinked chitosan thin film for all 
thickness is $\mathrm{n}(1.540 \pm 0.005)$ and $\mathrm{k}(0.015 \pm 0.002)$. The thin film with concentration $0.40 \mathrm{~g}$ chitosan in acetic acid coated on second set of gold film $(\sim 50 \mathrm{~nm})$ shows the best SPR curve with narrowest and lowest reflectivity dip. This information will be used in our future research for SPR optical sensor.

\section{ACKNOWLEDGEMENT}

The researchers would like to thank the Malaysian Government for the fund support through SAGA. The laboratory facilities provided by the Department of Physics, Faculty of Science, University Putra Malaysia, are also acknowledged.

\section{REFERENCES}

Bhumkar, D.R. and V.B. Pokharkar, 2006. Studies on effect of $\mathrm{pH}$ on cross-linking of chitosan with sodium tripolyphosphate: A technical note. AAPS Pharm. Sci. Tech., 7: 138-143. DOI: 10.1208/pt070250

Hoffmann, A., N. Kroo, Z. Lenkefi and Z. Szentirmay, 1996. A high precision ATR study of surface Plasmon mediated reflectance in noble metal films. Surface Sci., 352-354: 1043-1046. DOI: 10.1016/0039-6028(95)01324-5

Homola, J., 2006. Surface plasmon resonance based sensors. 1st Edn., Springer, New York, ISBN-10: 3540339183, pp: 251.

Homola, J., S.S. Yee and G. Gauglitz, 1999. Surface plasmon resonance sensors: Review. Sens. Actuators B. Chem., 54: 3-15. DOI: 10.1016/S09254005(98)00321-9
Kurihara, K. and K. Suzuki, 2002. Theoretical understanding of an absorption-based surface Plasmon resonance sensor based on Kretchmann's theory. Anal. Chem., 74: 696-701. DOI: 10.1021/ac010820

Liedberg, B., C. Nylander and I. Lundstrom, 1983. Surface plasmon resonance for gas detection and biosensing. Sens. Actuators, 4: 299-304. DOI: 10.1016/0250-6874(83)85036-7

Mendelez, J., R. Carr, D. Bartholomew, H. Taneja and S. Yee et al., 1997. Development of a surface Plasmon resonance sensor for commercial applications, Sens. Actuators B. Chem., 39: 375-379. DOI: 10.1016/S0925-4005(97)80237-7

Sadowski, J.W., J. Lekkala and I. Vikholm, 1994. Biosensors based on surface plasmons excited in non-noble metals. Biosensors Bioelectrics 6: 439-444. DOI: 10.1016/0956-5663(91)87009-Z

Shauer, C.L., M.S. Chen, M. Chatterley, K. Eisemann and E.R. Welsh et al., 2003. Color changes in chitosan and poly (allyl amine) films upon metal binding. Thin Solid Films, 434: 250- 257. DOI: 10.1016/S0040-6090(03)00055-5

Berger, C.E.H., R.P.H. Kooyman and J. Greve, 1999. Surface plasmon propagation near an index step. Optics Communi., 167: 183-189. DOI: 10.1016/S0030-4018(99)00309-0

Yusmawati, W.Y.W., H.P. Chuah and W.M.M. Yunus, 2007. Optical properties and sugar content determination of commercial carbonated drinks using surface plasmon resonance. Am. J. Applied Sci., 4: 1-4. DOI: 10.3844/ajassp.2007.1.4 